TRABAJOS ORIGINALES

\title{
Modelamiento predictivo, distribución geográfica y estado de conservación de Tomopeas ravus Miller, 1900 (Chiroptera, Molossidae)
}

\section{Predictive modeling, geographic distribution and conservation status of Tomopeas ravus Miller, 1900 (Chiroptera, Molossidae)}

\section{Christian R. Loaiza Salazar ${ }^{1 *}$ y Víctor Pacheco Torres ${ }^{1,2}$}

\author{
1 Universidad Nacional Mayor de San Marcos, Departamento de Mastozoología, Museo de Historia Natural, , Av. Arenales 1256, Jesús María, Lima, Perú. \\ 2 Universidad Nacional Mayor de San Marcos, Instituto de Ciencias Biológicas “Antonio Raimondi”, Facultad de Ciencias Biológicas, Lima, Perú. \\ * Autor para correspondencia \\ Email Christian Loaiza: christian.loaiza@unmsm.edu.pe \\ Email Víctor Pacheco: vpachecot@unmsm.edu.pe
}

\begin{abstract}
Resumen
Tomopeas ravus, el murciélago de orejas romas, se conoce solo de 13 localidades confirmadas en Perú y actualmente se encuentra listada como En Peligro (B2 ab (iii)) por la UICN. La distribución, extensión de ocurrencia y el área de vida de esta especie no han sido esclarecidos completamente. Presentamos el primer modelo predictivo basado en los registros conocidos y el primer acercamiento geográfico para la especie. Basados en los 13 registros confirmados se construyó un modelo de hábitat de máxima entropía determinando previamente las variables ambientales de mayor importancia para el modelo. Se estimó un índice predictivo aceptable (0.970) para el modelo final. Se determinó las zonas de mayor probabilidad de ocurrencia para esta especie y se estimó el área de distribución mediante el método del polígono mínimo convexo (PMC). El área prioritaria más importante identificada para la conservación de esta especie se sitúa al norte de Perú, entre los Departamentos de Tumbes y Piura. Se recomienda utilizar el presente modelo como herramienta útil para futuras evaluaciones de la conservación de esta especie.
\end{abstract}

Palabras clave: Perú; Molossidae; En Peligro; Modelo predictivo; Ecorregión tumbesina.

\section{Abstract}

Tomopeas ravus, known as blunt - eared bat, is known only from thirteen confirmed localities in Peru and is currently listed as Endangered (B2 ab (iii)) by the IUCN. The distribution, occurrence extension and home range of this species is not have been completely clarified. We presents the first predictive model based on the known records and the first geographic approach to this species. On the basis of thirteen confirmed records we built a habitat model of maximum entropy determining previously the most important environmental variables for the model. An acceptable predictive index (0.970) was obtained for the final model. We determined the areas of major probability of occurrence for this species and felt the geographic distribution through the method of the polygon mínimum convex (PMC). The priority area identified for the conservation of this species is located in the northern of Peru, between the Departments of Tumbes and Piura. We recommended to use the present model as a useful tool for future evaluations of conservation of this species.

Keywords: Peru; Molossidae; Endangered; Predictive model; Tumbesian ecoregion.

Citación:

Loaiza Salazar. C.R. \& V. Pacheco Torres. 2017. Modelamiento predictivo, distribución geográfica y estado de conservación de Tomopeas ravus Miller, 1900 (Chiroptera, Molossidae). Revista peruana de biología 24(2) 193 - 198 (Julio 2017). doi: http://dx.doi.org/10.15381/rpb.v24i2.13495
Información sobre los autores:

CLS, realizó el diseño experimental, elaboración del mapa y redacción del artículo. VPT aportó con la base de datos del Museo, revisión del artículo, sugerencias y comentarios sobre la biogeografía y distribución de la especie. CRL-S, VPT: revisaron y aprobaron el manuscrito.

Los autores no incurren en conflictos de intereses. 


\section{Introducción}

El bosque seco de Tumbes-Piura es una ecorregión de bosque seco tropical situada en el noroccidente de Perú (Tumbes, Piura, Lambayeque y Cajamarca) y el suroccidente del Ecuador (Azuay, Guayas, Loja y El Oro) (Pennington et al. 2006). Esta ecorregión en conjunto con el bosque seco del Marañón y el bosque seco ecuatoriano conforman la gran región tumbesina reconocida por algunos autores como bosque seco ecuatorial, región de endemismo tumbesina o también como bosque seco ecuatorial tumbesino (Brack Egg 1986, Best \& Kessler 1995). Otros autores como Morrone (2017) han propuesto dividir el bosque seco ecuatorial bajo otra clasificación agrupada en dos regiones biogeográficas: 1) Provincia del Ecuador occidental, situada en la zona centro-noroccidente del Ecuador y suroccidente de Colombia, y 2) Provincia Ecuatoriana, situada al sur de Ecuador y norte del Perú.

La región tumbesina debido a su ubicación estratégica y la influencia de ciertos factores ecológicos, geográficos y ambientales, posee un elevado nivel de endemismos, lo cual puede ser reconocido en casi todos los grupos de flora y fauna presentes en dicha región (Best \& Kessler 1995). Dentro del grupo de los mamíferos, destacan tres géneros (Amorphochilus, Platalina y Tomopeas) y cerca de 20 especies las que se encuentran parcial o completamente distribuidas dentro de esta región (Tuttle 1970, Koopman 1978, Aguilar 1994, Pacheco et al. 2007, Baker et al. 2009, Pacheco et al. 2009, Gregorio \& Almeida 2010, Tirira et al. 2011, Loaiza 2013, Pinto et al. 2013, Flores-Quispe et al. 2015).

Tomopeas ravus es una especie de quiróptero (Molossidae) monotípico y endémico del Perú (Barkley 2007, Pacheco et al. 2009). Habita en la región costera desértica del norte y centro del país, con excepción de algunos registros en zonas altoandinas en la región de Cajamarca, al norte de Perú (Miller 1900, De la Puente 1951, Aellen 1965, Davis 1970, Velazco et al. 2013, Zamora et al. 2014). Nuevos registros extienden su área de distribución hacia el sur de Perú, en la región de Arequipa (Pari et al. 2015). Su rango altitudinal se sitúa entre $0-2000 \mathrm{~m}$ de altitud, aunque existen reportes de su presencia por encima de los 2000 metros (Velazco et al. 2013). Es una especie típica de las zonas semiáridas de la costa peruana. Está considerada como una especie en peligro (EN) y amenazada por ciertos factores como la agricultura, la expansión urbana y otros tipos de factores antropogénicos (Zamora et al. 2014, Velazco 2016).

Nuestro objetivo fue presentar un análisis zoogeográfico mediante el primer modelo predictivo basado en los registros conocidos y el primer acercamiento geográfico/distributivo para esta especie.

\section{Material y métodos}

Modelamiento distributivo.- Como paso previo al modelamiento se procedió a descargar un conjunto de variables ambientales del world clim (www.worldclim.org), las cuales comprenden diferentes tipos de factores bioclimáticos en formato raster. Se descargó el archivo de bioclima 30s en formato ESRI grid $(-1$ $\mathrm{km}$ ) y se realizó un recorte de las variables para la república del Perú, con el fin de contar con un juego de variables ambientales específicamente diseñado para Perú.

Para obtener la distribución potencial de esta especie se construyó una base de datos, la cual contó con 13 registros obtenidos en base a la revisión de publicaciones y de la base de datos del Departamento de Mastozoología del Museo de Historia Natural de San Marcos (MUSM) (Tabla 1). Para analizar la distribución potencial se utilizó el programa Maxent (Phillips et al. 2006, Phillips \& Dudik 2008) y se utilizó la misma metodología usada en algunas publicaciones anteriores (Loaiza \& Roque 2016, Loaiza 2017), la cual consiste en desarrollar 10 réplicas para el modelo, con el $25 \%$ de los registros usados como test data, 500 iteraciones y 10000 puntos de respaldo background points. Esta metodología se aplicó para hacer un jackknife y determinar las variables ambientales que mejor contribuyen con el modelamiento. Luego de registrar las variables ambientales con menor valor de contribución con el modelamiento, se corrió nuevamente el programa y se seleccionó como archivo de salida output un

Tabla 1: Registros confirmados de Tomopeas ravus utilizados en el modelamiento potencial del hábitat. Ubicación biogeográfica según el tipo de ecorregión de la WWF y tipo de formación vegetal, de acuerdo al sistema de cobertura vegetal establecido por el Ministerio del Ambiente del Perú (MINAM) / 2015.

\begin{tabular}{|c|c|c|c|c|}
\hline Localidades & Latitud & Longitud & Ecorregión (WWF) & Formación vegetal \\
\hline Yayán (Cajamarca) & $07^{\circ} 18^{\prime}$ & $79^{\circ} 19^{\prime}$ & Tumbes - Piura & Matorral arbustivo \\
\hline Chosica (Lima) & $11^{\circ} 54^{\prime}$ & $76^{\circ} 42^{\prime}$ & Desierto de Séchura & Cardonal \\
\hline Mallares (Piura) & $04^{\circ} 51^{\prime} 50.0^{\prime \prime}$ & $80^{\circ} 46^{\prime} 23.8^{\prime \prime}$ & Tumbes - Piura & Matorral arbustivo \\
\hline Laderas del cerro Amotape (Piura) & $04^{\circ} 45^{\prime} 57.0^{\prime \prime}$ & $80^{\circ} 59^{\prime} 56.0^{\prime \prime}$ & Tumbes - Piura & Bosque seco de piedemonte \\
\hline Monte Grande, 14 km al norte de Talara (Piura) & $04^{\circ} 27^{\prime} 32.0^{\prime \prime}$ & $81^{\circ} 02^{\prime} 46.0^{\prime \prime}$ & Tumbes - Piura & Bosque seco tipo sabana \\
\hline Pariñas, 7 km al norte de Talara (Piura) & $04^{\circ} 30^{\prime} 46.0^{\prime \prime}$ & $81^{\circ} 08^{\prime} 03.0^{\prime \prime}$ & Tumbes - Piura & Bosque seco tipo sabana \\
\hline Quebrada Hondo, 14 km al norte de Talara (Piura) & $04^{\circ} 26^{\prime} 58.0^{\prime \prime}$ & $81^{\circ} 08^{\prime} 03.0^{\prime \prime}$ & Tumbes - Piura & Bosque seco de colina baja \\
\hline Cerro La Vieja (Lambayeque) & $06^{\circ} 15^{\prime} 48.0^{\prime \prime}$ & $79^{\circ} 41^{\prime} 00.0^{\prime \prime}$ & Tumbes - Piura & Bosque seco de piedemonte \\
\hline Fondo, 14 km al norte de Talara (Piura) & $04^{\circ} 26^{\prime} 25.0^{\prime \prime}$ & $81^{\circ} 10^{\prime} 31.0^{\prime \prime}$ & Tumbes - Piura & Matorral arbustivo \\
\hline Quebrada Pariñas (Piura) & $04^{\circ} 31^{\prime} 41.2^{\prime \prime}$ & $81^{\circ} 12^{\prime} 09.0^{\prime \prime}$ & Tumbes - Piura & Bosque seco ribereño \\
\hline $12 \mathrm{~km}$ al norte de Olmos (Lambayeque) & $05^{\circ} 50^{\prime} 30.0^{\prime \prime}$ & $79^{\circ} 47^{\prime} 00.0^{\prime \prime}$ & Tumbes - Piura & Bosque seco tipo sabana \\
\hline Quebrada de San Juan (Ica) & $13^{\circ} 42^{\prime} 14.0^{\prime \prime}$ & $75^{\circ} 38^{\prime} 47.0^{\prime \prime}$ & Desierto de Séchura & Cardonal \\
\hline Valle del Alto Molina (Arequipa) & $15^{\circ} 55^{\prime} 56.8^{\prime \prime}$ & $73^{\circ} 07^{\prime} 49.9^{\prime \prime}$ & Desierto de Séchura & Desierto costero \\
\hline
\end{tabular}


Tabla 2: Resultados de las áreas predictivas para los valores de corte acumulativo 1, 5, 10 y valor de corte mínimo generados para el modelamiento.

\begin{tabular}{cc}
\hline $\begin{array}{c}\text { Valores de corte } \\
\text { acumulativo }\end{array}$ & Modelamiento predictivo \\
\cline { 2 - 2 } 1 & Fraccional de área predictiva \\
5 & 0.179 \\
10 & 0.11 \\
Mínimo & 0.086 \\
\hline
\end{tabular}

modelo en formato grid logístico para poder determinar las áreas con la mayor probabilidad de ocurrencia para esta especie.

Como análisis estadístico, se evaluaron los test dependiente e independiente, usando para ellos como umbral de corte los valores de P en 1, 5 y 10 bajo el área de curva AUC (Tabla 2) (Mateo et al. 2011). Para el umbral de corte, se seleccionó la opción para el mínimo de presencia de los puntos de entrenamiento Minimum training presence, la cual permite obtener modelos con mayor nivel de precisión.

Área de distribución.- Para estimar el área de distribución geográfica se utilizó una herramienta disponible en ArcGis llamada Xtools Pro, la cual permite crear polígonos en base a los registros de una especie y además, estimar el área de distribución en $\mathrm{km}^{2}$. Debido a la concentración de registros claramente diferenciados al norte y en la zona centro-sur del Perú, se crearon dos polígonos por separado a fin de poder delimitar el área de distribución respectiva de esta especie en base a sus registros. Los mapas finales fueron realizados con el programa ArcGis 10.3

\section{Resultados}

Modelamiento distributivo.- El modelamiento realizado en Maxent para esta especie tuvo un nivel de aceptación bastante óptimo. El valor de sensibilidad obtenido en el test data fue de 0.975 , lo cual indica que el $97.5 \%$ de las localidades observadas estarían incluidas en la predicción (datos de presencia). El valor de AUC obtenido en el modelamiento final fue de 0.970 (97\%), lo cual indica un nivel de confianza igualmente aceptable. El grado de predicción al azar obtenido fue de 0.5 , lo cual indica que el modelo obtenido no es diferente de clasificar los casos al azar, sin embargo, los valores de sensibilidad obtenidos en el análisis permiten tener un grado de aceptación bastante óptimo del modelamiento.

De las variables utilizadas, siete variables con valores superiores al $1 \%$ contribuyeron con el modelamiento, lo cual representa el $98.1 \%$ de la varianza explicada (Tabla 3). Las variables ambientales con mayor influencia para la distribución de $T$. ravus fueron: Estacionalidad de la temperatura (29.6\%), Temperatura media del cuatrimestre más lluvioso (22\%), Precipitación del periodo más seco $(25.8 \%)$ y Estacionalidad de la precipitación (11.7\%). Otras variables de mayor y menor importancia se señalan en la Tabla 3.

Distribución y biogeografía.- En base al modelamiento realizado se elaboró un mapa de distribución potencial (Fig. 1), el cual representa las zonas con la mayor probabilidad de presencia y permite establecer a la vez las áreas con la mayor importancia en términos de conservación.

Tabla 3: Porcentaje de contribución (\% C) e importancia de la permutación (PI) de cada variable para el modelamiento logístico y potencial.

\begin{tabular}{|c|c|c|c|}
\hline \multirow{2}{*}{ Variables } & \multirow{2}{*}{ Descripción } & \multicolumn{2}{|c|}{ Modelamiento } \\
\hline & & $\% \mathrm{C}$ & PI \\
\hline bio_1 & Temperatura media anual & 0 & 0 \\
\hline bio_2 & Oscilación diurna de la temperatura & 0 & 0 \\
\hline bio_3 & Isotermalidad & 0.3 & 0.4 \\
\hline bio_4 & Estacionalidad de la temperatura (\%) & 29.6 & 0 \\
\hline bio_5 & Temperatura máxima del período más cálido & 5.2 & 0 \\
\hline bio_6 & Temperatura mínima del período más frío & 0 & 0 \\
\hline bio_7 & Oscilación anual de la temperatura & 0.1 & 0 \\
\hline bio_8 & Temperatura media del cuatrimestre más lluvioso & 22 & 38.1 \\
\hline bio_9 & Temperatura media del cuatrimestre más seco & 0 & 0 \\
\hline bio_10 & Temperatura media del cuatrimestre más cálido & 0 & 0 \\
\hline bio_11 & Temperatura media del cuatrimestre más frío & 0 & 0 \\
\hline bio_12 & Precipitación anual (mm) & 1.4 & 49.1 \\
\hline bio_13 & Precipitación del período más lluvioso & 0 & 0 \\
\hline bio_14 & Precipitación del período más seco & 25.8 & 11.8 \\
\hline bio_15 & Estacionalidad de la precipitación (\%) & 11.7 & 0.6 \\
\hline bio_16 & Precipitación del cuatrimestre más lluvioso & 1 & 0 \\
\hline bio_17 & Precipitación del cuatrimestre más seco & 0.3 & 0 \\
\hline bio_18 & Precipitación del cuatrimestre más cálido & 2.4 & 0 \\
\hline bio_19 & Precipitación del cuatrimestre más frío & 0 & 0 \\
\hline Total & & 99.8 & - \\
\hline
\end{tabular}




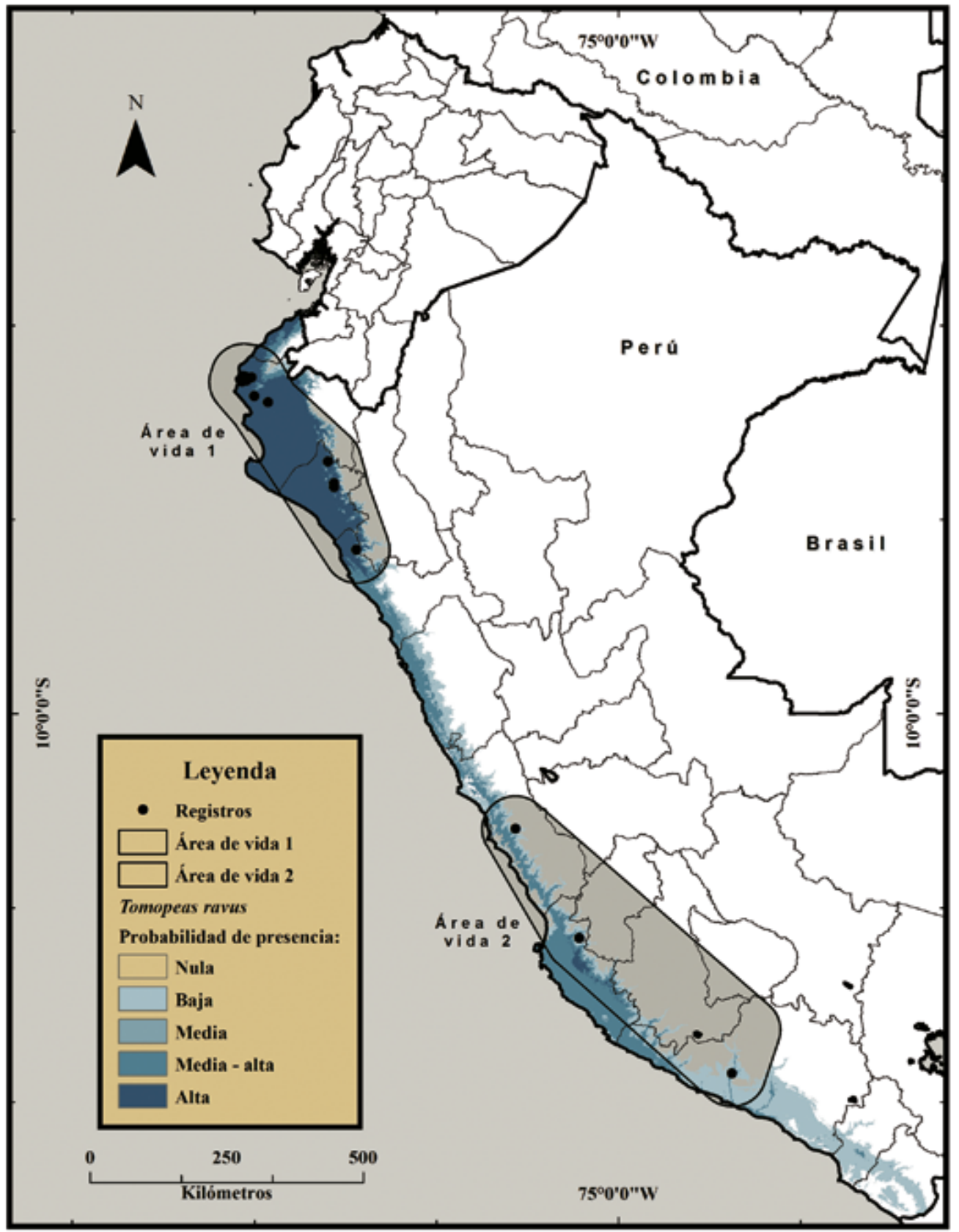

Figura 6: Mapa de distribución geográfica y probabilidad de presencia de Tomopeas ravus.

Los polígonos establecidos al norte $\left(65944.5 \mathrm{~km}^{2}\right)$ y en la zona centro-sur de Perú $\left(119027.3 \mathrm{~km}^{2}\right)$, dieron un valor total de $122152.5 \mathrm{~km}^{2}$ (Fig. 1), lo cual representa un área importante de extensión distributiva.

\section{Discusión}

En base al modelamiento predictivo, se establece que el área con la mayor probabilidad de presencia para $T$. ravus (Fig. 1), se ubica al norte de Perú, entre los departamentos de Tumbes, Piura y Lambayeque. El área que corresponde al segundo polígono, entre los departamentos de Lima, Ica y Arequipa, muestra una probabilidad de presencia media-baja, lo cual puede estar relacionado con la falta de estudios o también debido a la poca efectividad obtenida con los métodos de muestreo tradicionales. Se establece que la región de Ica es la que reúne las mejores con- diciones de hábitat (ecológicas/ambientales) como para albergar poblaciones adecuadas de esta especie.

La distribución que presenta $T$. ravus tiene bastante similitud con la distribución de otras especies de molósidos como Promops davisoni (Flores-Quispe et al. 2015) y algunas especies de otros grupos como Artibeus fraterculus, Platalina genovensium y Glossophaga valens (Ruelas et al. en prensa), lo cual sugiere la existencia de un patrón distributivo común para estas especies. Este patrón distributivo ya ha sido observado en otros grupos de flora y fauna (Loaiza 2013), sugiriendo que la capacidad de dispersión está relacionada con la ecología de las especies y los mecanismos usados para su dispersión (Ruelas et al. en prensa).

La ecorregión conocida como Bosque seco de Tumbes-Piura es la que concentra la mayor cantidad de registros para esta 
especie (76.9\%), tanto históricamente como en años recientes, por lo cual los esfuerzos de conservación se deben enfocar y ser prioritarios en dicha ecorregión, tomando en cuenta los registros de las localidades conocidas.

Es probable que la región de Arequipa, especialmente algunas zonas como el valle del Alto Molina, la cuenca del río Ocońa y la subcuenca de Cotahuasi, además de otras zonas parecidas, presenten las condiciones de hábitat idóneo para ofrecer refugios clave para esta especie, por lo cual se recomienda incentivar los esfuerzos de estudio a fin de ubicar y establecer los refugios y dormideros específicos de esta especie con fines de conservación.

Se considera que el bajo número de registros de $T$. ravus al centro y sur de Perú se debe a la falta de estudios, la poca probabilidad de presencia de esta especie, especialmente al sur del Perú y probablemente, debido a lo reducida que resulta el área de distribución de esta especie en dichas regiones a diferencia del norte de Perú, además de que los registros de Lima, Arequipa e Ica, se encuentran dentro de la ecorregión conocida como Desierto de Séchura, en las formaciones vegetales de Cardonal y Desierto costero, lo cual sugiere que las condiciones ambientales y ecológicas en dichas región, al ser distintas a las condiciones existentes en el norte de Perú, probablemente deben influir en la distribución de la especie.

Finalmente, otro aspecto que influiría en la distribución y abundancia de $T$. ravus, sobre todo en el caso de Lima, debe tener relación con la expansión de las áreas urbanas y la consecuente modificación de las pocas áreas de bosque natural, lo cual seguramente debe estar afectando e influyendo de forma negativa en la distribución de esta especie.

Estado de conservación.- Las evaluaciones realizadas en años anteriores sobre el estado de conservación de esta especie han determinado que la reducción y fragmentación del hábitat, así como la alteración de sus refugios diurnos por actividades antrópicas, han influido gravemente provocando una reducción del tamańo de sus poblaciones, siendo considerada actualmente como una especie en peligro (EN) según el criterio B2 ab (ii, iii) (Velazco 2016). Sin embargo, esta hipótesis está motivada por la rareza de esta especie y los pocos registros que se tiene de ella, además de que hasta la fecha no se conoce de ningún estudio que haya determinado el tamańo exacto de sus poblaciones.

El modelamiento obtenido en este trabajo señala una probabilidad de presencia media-alta entre las regiones de Ancash y La Libertad, la cual se extiende hasta llegar al norte de Lima, empezando a reducirse de forma progresiva conforme se extiende hacia el sur de Perú. El registro de esta especie en la región de Ancash y La Libertad permitiría evaluar y proponer mejores alternativas para la conservación de esta especie, así como también establecer o descartar una conectividad entre las poblaciones situadas al norte y sur de Perú.

A pesar de que $T$. ravus está considerada como una especie endémica del Perú, existe cierta probabilidad de que su área de distribución se extienda hacia el suroccidente del Ecuador, específicamente hacia la provincia de Loja y dado los recientes hallazgos y nuevos registros de algunas especies como $A$. schnablii y L. hesperia (Tirira et al. 2011, Tirira 2012), es muy probable que su área de distribución sea mucho más amplia de lo que se considera actualmente.
El área situada al norte del Perú, pese a tener una mayor concentración de registros de esta especie, es la que mayores amenazas de conservación presenta, debido a la alteración y perturbación de hábitat, lo cual unido al desconocimiento que se tiene por parte del ser humano, puede poner en riesgo los pocos refugios disponibles para esta especie.

Es necesario desarrollar campañas de educación ambiental enfocadas en la conservación de esta especie, así como también reiterar en la urgencia de establecer alguna normativa ambiental validada por el Ministerio del Ambiente del Perú para declarar como áreas protegidas las zonas de mayor distribución de esta especie.

Se recomienda también implementar estudios especializados en determinar el área de vida en base a registros con equipos acústicos a fin de conocer su comportamiento, sitios de forrajeo y refugios diurnos, lo cual es vital para la conservación de esta especie.

\section{Agradecimientos}

Este trabajo representa el primer esfuerzo de una serie de artículos enfocados en analizar la distribución y el estado de conservación de las especies de mamíferos endémicos de los bosques secos del sur de Ecuador y norte del Perú. Los autores expresan su agradecimiento a Richard Cadenillas, quien revisó este manuscrito y aportó con sugerencias para el mejoramiento del mismo. Dos revisores anónimos contribuyeron con sus observaciones y sugerencias para mejorar sustancialmente el manuscrito inicial. Se agradece también al Departamento de Mastozoología del Museo de Historia Natural (MUSM) de la Universidad Nacional Mayor de San Marcos por facilitar la base de datos de la colección de murciélagos.

\section{Literatura citada}

Aellen V. 1965. Sur une petite collection de chiropteres du nordouest du Perou. Mammalia 29: 563-571. doi: http://dx.doi. org/10.1515/mamm.1965.29.4.563

Aguilar P. 1994. Características faunísticas del norte del Perú. Arnaldoa 2 (1): 77-102.

Baker R.J., M.M. McDonough, V.J. Swier, P.A. Larsen, J.P. Carrera \& L.K. Ammerman. 2009. New species of bonneted bat, genus Eumops (Chiroptera: Molossidae) from the lowlands of western Ecuador and Peru. Acta Chiropterologica 11 (1): 1-13. doi: http://dx.doi.org/10.3161/150811009X465659

Barkley L.J. 2007. Subfamily Tomopeatinae Miller, 1907. Pp. 439440, in: Mammals of South America (A. L. Gardner, ed.). Vol. 1. Marsupials, xenarthrans, shrews, and bats. University of Chicago Press, Chicago, Illinois. 669 pp.

Best B.J. \& M. Kessler. 1995. Biodiversity and conservation in Tumbesian Ecuador and Peru. Cambridge, U. K. Birdlife International. 218 pp.

Brack-Egg, E. 1986. Las ecorregiones del Perú. Boletín de Lima, 44: $57-70$.

Davis W.B. 1970. Tomopeas ravus Miller (Chiroptera). Journal of Mammalogy 51 (2): 244-247. doi: http://dx.doi. org/10.2307/1378474

De la Puente J. 1951. Estudio monográfico de los quirópteros de Lima y sus alrededores. Publicaciones del Museo de Historia Natural "Javier Prado". Universidad Nacional Mayor de San Marcos. Serie Zoología 7: 1-48.

Díaz M.M., S. Solari, L.F. Aguirre, L.M. Aguiar \& R.M. Barquez. 2016. Clave de identificación de los murciélagos de Sudamérica. Publicación Especial No. 2. Programa de Conservación de los Murciélagos de Argentina. 160 pp. 
Flores-Quispe M., G. Calizaya-Mamani, V. Pacheco \& G. AragónAlvarado. 2015. Distribution of Promops davisoni Thomas, 1921 (Chiroptera: Molossidae) in Peru with a new record and southward range extension. Check List 11 (2): 1-7. doi: http://dx.doi.org/10.15560/11.2.1573

Gregorio R. \& E. Almeida. 2010. Revalidation of Promops davisoni Thomas (Molossidae). Chiroptera Neotropical 16 (1): 648-660.

Koopman K.F. 1978. Zoogeography of Peruvian bats, with special emphasis on the role of the Andes. American Museum Novitates. No. 2651: 1-40.

Loaiza C.R. 2013. The Tumbesian center of endemism: biogeography, diversity and conservation. Bulletin of the Systematic and Evolutionary Biogeographical Association 6: 4-10.

Loaiza C.R. \& J. Roque. 2016. Revalidación taxonómica y distribución potencial de Armatocereus brevispinus Madsen (Cactaceae). Revista Peruana de Biología 23 (1): 35-41. doi: http://dx.doi. org/10.15381/rpb.v23i1.11831

Loaiza C.R. 2017. Área de vida, distribución potencial y estado de conservación de Espostoa frutescens Madsen, 1989 (Cactaceae). Ecología Aplicada 16 (1): 1-7. doi: http://dx.doi. org/10.21704/rea.v16i1.897

Mateo R.G., A.M. Felicísimo \& J. Muñoz. 2011. Modelos de distribución de especies: una revisión sintética. Revista Chilena de Historia Natural 84: 217-240. doi: http://dx.doi. org/10.4067/S0716-078X2011000200008

Miller G.S. 1900. A new bat from Perú. Annals and Magazine of Natural History 7 (6): 570-574. doi: http://dx.doi. org/10.1080/00222930008678426

Ministerio del Ambiente. 2015. Mapa nacional de cobertura vegetal: memoria descriptiva. Dirección General de Evaluación, Valoración y Financiamiento del Patrimonio Natural Lima. 105 pp.

Morrone J.J. 2017. Neotropical biogeography, regionalization and evolution. CRC Press, Taylor \& Francis Group, Boca Ratón, Florida, $282 \mathrm{pp}$.

Pacheco V., R. Cadenillas, S. Velazco, E. Salas \& U. Fajardo. 2007. Noteworthy bat records from the Pacifical Tropical rainforest region and adjacent dry forest in northwestern Peru. Acta Chiropterologica 9 (2): 409-422. doi: http://dx.doi. org/10.3161/1733-5329(2007)9[409:NBRFTP]2.0.CO;2

Pacheco V., R. Cadenillas, E. Salas, C. Tello \& H. Zeballos. 2009. Diversidad y endemismo de los mamíferos del Perú. Revista Peruana de Biología 16 (1): 5-32. doi: http://dx.doi. org/10.15381/rpb.v16i1.111

Pari A., K. Pino, C.E. Medina, E. López \& H. Zeballos. 2015. Murciélagos de Arequipa, Historia Natural y Conservación. Arequipa, Perú. 179 pp.
Pennington R.T., G.P. Lewis \& J.A. Ratter. 2006. Neotropical savannas and seasonally dry forests: plant diversity, biogeography, and conservation. The Systematics Association, Special Volumes. No. 69. 504 pp.

Phillips S.J., R.P. Anderson \& R.E. Schapire. 2006. Maximum entropy modeling of species geographic distributions. Ecological Modeling 190: 231-259. doi: http://dx.doi.org/10.1016/j. ecolmodel.2005.03.026

Phillips S.J. \& M. Dudík. 2008. Modeling of species distributions with Maxent: new extensions and a comprehensive evaluation. Ecography 31: 161-175. doi: http://dx.doi.org/10.1111/ j.0906-7590.2008.5203.x

Pinto C.M., M.R. Marchán-Rivadeneira, E. Tapia, J.P. Carrera \& R.J. Baker. 2013. Distribution, abundance and roosts of the fruit bat Artibeus fraterculus (Chiroptera: Phyllostomidae). Acta Chiropterologica 15 (1): 85-94. doi: http://dx.doi. org/10.3161/150811013X667885

Ruelas D., V. Pacheco \& O. Centty. En prensa. Taxonomic status of Glossophaga soricina valens (Chiroptera: Phyllostomidae). Acta Chiropterologica.

Tirira D.G., S.F. Burneo, C.E. Boada \& S.E. Lobos. 2011. Mammalia, Chiroptera, Phyllostomidae, Lonchophylla hesperia G. M. Allen, 1908: Second record of the western nectar bat in Ecuador after 70 years. Check List 7 (3): 315-318. doi: http://dx.doi.org/10.15560/7.3.315

Tirira D.G. 2012. Murciélagos del Ecuador: una referencia geográfica, taxonómica y bibliográfica. Pp. 235 - 326, en: Investigación y conservación sobre murciélagos en el Ecuador (D. G. Tirira y S. F. Burneo, eds.). Pontificia Universidad Católica del Ecuador, Fundación Mamíferos y Conservación y Asociación Ecuatoriana de Mastozoología. Publicación especial sobre los mamíferos del Ecuador 9. Quito.

Tuttle M.D. 1970. Distribution and zoogeography of Peruvian bats, with comments on natural history. The University of Kansas Science Bulletin. Vol. 49, pp. 45-86.

Velazco P.M. 2016. Tomopeas ravus. The IUCN Red List of Threatened Species 2016. <www.iucnredlist.org>. Downloaded on 09 December 2016.

Velazco P.M., R. Cadenillas, O. Centty, L. Huamaní \& H. Zamora. 2013. New records of Platalina genovensium (Chiroptera, Phyllostomidae) and Tomopeas ravus (Chiroptera, Molossidae). Mastozoología Neotropical 20 (2): 425-434.

Zamora H., C. Medina, A. Escobar, Y. Arteaga, R. Cadenillas \& P.M. Velazco. 2014. New distributional record of the rare endemic Peruvian Tomopeas ravus Miller, 1900 (Chiroptera, Molossidae, Tomopeatinae). Mammalia 78 (2): 257-260. doi: http://dx.doi.org/10.1515/mammalia-2013-0007 